\title{
Stimulus factors in auditory identification learning'
}

\author{
A. M. BARGH AND J. R. LEVINE ${ }^{2}$ \\ MICHIGAN STATE UNIVERSITY
}

\begin{abstract}
Abstraet
Paired-associate learning with Morse code signals as stimuli was facilitated as the amount of prior stimulus familiarization was increased. High interstimulus similarity of the auditory signals retarded paired-associate learning, especially during the initial trials. However, interstimulus similarity did not interact significantly with the effect of stimulus pretraining.
\end{abstract}

\section{Problem}

How does prior acquaintance with material to be learned influence learning? Gibson (1940) proposed that procedures that increase differentiation among the stimuli would reduce the amount of generalization and thereby reduce errors. A number of studies confirm the value of stimulus familiarizarion (SF) when visual or configurational stimuli are used (Arnoult, 1957). Generally negative results have been found when printed verbal material has been used as stimuli (e.g., Underwood \& Schultz, 1960) although the issue is not clearly resolved.

Only one published study has used aural stimuli-Schultz \& Martin (1963), who found SF to generally facilitate the learning of trigram-number pairs. Experimentally produced familiarity with nonverbal auditory stimuli has not been reported.

All various theoretical explanations for SF effects would agree that the degree of stimulus similarity is a highly important variable. Yet, no published study has specifically related SF to intralist stimulus similarity in any sensory modality. However, Rothkopf (1957) has scaled the similarity of Morse code signals by a psychophysical procedure. Thus, sets of auditory stimuli with known degrees of similarity can be assembled for familiarization work.

\section{Method and Results}

The Ss were 120 college student volunteers, 20 per experimental group, run in small groups of 4-5 each. The International Morse code signals used were transmitted at a rate of 12-15 words per minute with an intensity of 85-95 db and a frequency of 925-935 cps. Two sets of eight signals were used. Four signals were common to both sets and four were unique to each set. The mean percent similarity (confusability) of the High set $(\mathrm{H})$ was 30 while the mean percent similarity of the Low set (L) was 11. The percent of interstimulus confusions for pairs of signals varied from 2 to 32 for the $L$ set and from 7 to 79 for the $H$ set.

SF training consisted of 0,20 , or 40 trials with each of the 8 signals sounded once during each trial at 4-sec. intervals. The attentional procedure was used-Ss were not required to make any overt responses to the signals but did know that the signals would be utilized later in the experiment.

In the paired-associate (PA) task Ss learned two-digit numbers as responses to the signals. The response numbers were as heterogeneous as possible. The entire set was written by each $\mathrm{S}$ on his own answer sheet before PA learning began and was continuously available. Thus, response learning per se was minimized and errors made could be directly attributed to either stimulus confusions or to failure to form associative bonds. Ss were told to guess if necessary. Virtually no omissions occurred.

Learning was by the anticipation method with a 4-4 sec. rate and a $6 \mathrm{sec}$. intertrial interval. Ss wrote their responses. A different random order of the stimuli was used on each of the 25 trials with the first trial a presentation trial only. SF and PA trials were presented by tape recorder. None of the Ss had had prior Morse code training of any kind.

The total number of correct responses during PA learning for the $L$ groups was $110.2,122.7$, and 129.6 for 0,20 , and 40 trials of SF respectively. The corresponding results for the $\mathrm{H}$ groups were 75.2, 91.4, and 101.3. The PA trials were divided into 8 practice stages of 3 trials each and an F test applied. The $\mathrm{F}$ ratios for $\mathrm{SF}$, similarity level, and the interaction of stage of practice by similarity level were all significant at the .01 level. Each of the three SF levels was significantly different by the $Q$ test from the other two levels $(p<.05)$. The interaction between SF and similarity level was clearly non-significant. The performance curves for the SF levels are shown in Fig. 1.

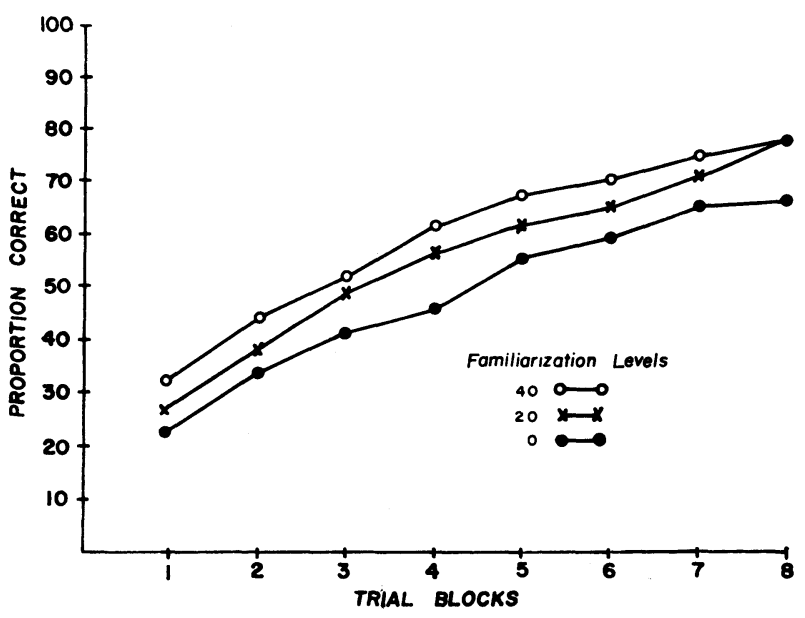

Fig. 1. Mean proportion correct for each stimulus familiarization level over blocks of three trials. 


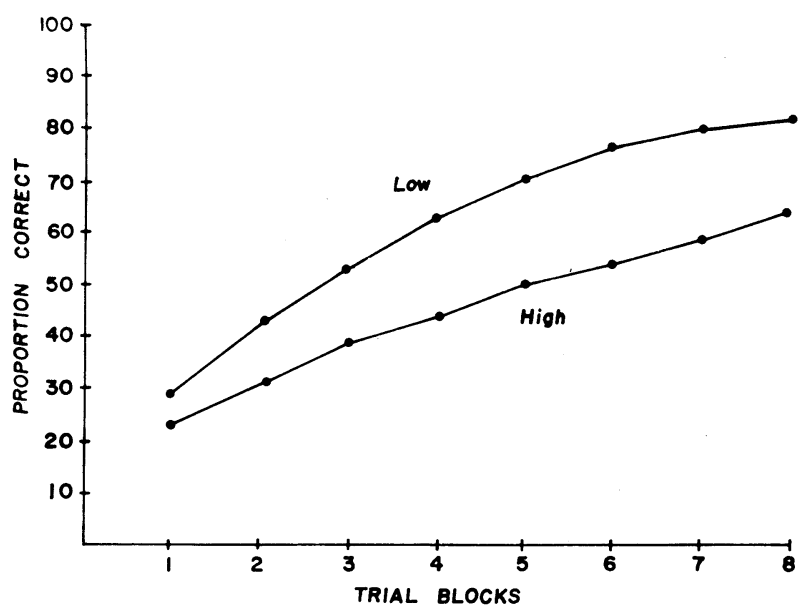

Fig. 2. Mean proportion correct on high and low similarity lists over blocks of three trials.

\section{Discussion}

Our experimental design did not use the so-called "irrelevant stimuli" control for warm-up effects because pretraining with such extraneous stimuli may produce unmeasurable negative transfer effects. If familarization here was only a warm-up experience, the SF groups should be facilitated only during the early trials. Figure 1 clearly shows that the group curves are parallel throughout.

A subsequent study directly tested for warm-up by introducing a $40 \mathrm{~min}$. delay (normal classroom activity) between SF and PA learning. The PA performance of the delay and non-delay groups (65 Ss per group) was virtually identical.

The faster learning of the groups with low interstimulus similarity is confirmatory of prior work with Morse code signals, verbal materials, and visual shapes. Figure 2 shows the performance curves for the similarity levels with SF ignored. The significant interaction between similarity level and stage of practice appears mainly due to a differential rate of improvement early on this task. Both $\mathrm{L}$ and $\mathrm{H}$ groups showed equivalent gains in proficiency during the later practice trials.

SF might be expected to become more beneficial as stimulus similarity is increased. If so, the difference in performance level between the $L$ groups and the $\mathrm{H}$ groups should decrease significantly as the amount of SF is increased. This L-H difference in total correct responses did decrease regularly from 35.0 to 31.3 to 28.3 as SF increased, but the trend was not significant.

The lack of significance of this trend does not represent a serious threat to predifferentiation hypotheses which stress confusions due to stimulus generalization. The intragroup differences were large, reducing the sensitivity of the statistical test. We are currently exploring methods for estimating and controlling such individual differences in auditory rhythmic ability.

Our results do show clearly the potential of Morse code signals for basic research in stimulus pretraining and auditory identification learning. The SF effects obtained were marked and persistent despite the relatively weak method of pretraining (attentional rather than paired-associate), the short series of test trials, and the wide intragroup variability.

\section{Referenees}

Arnoult, M. D. Stimulus differentiation: Some generalizations and hypotheses. Psychol. Bull., 1957, 54, 339-350.

Gibson, E. J. A systematic application of the concepts of generalization and differentiation to verbal learning. Psychol. Rev., 1940, 47, 196-229.

Rothkopf, E. Z. A measure of stimulus similarity and errors in some paired-associate learning tasks. J. exp. Psychol., 1957, 53, 94-101.

Schultz, R. W., \& Martin, E. Aural paired-associate learning: Stimulus familiarization; response familiarization; and prounciability. J. verbal Learn. verbal Behav., 1964, 3, 139-145.

Underwood, B. J., \& Schultz, R. W. Meaningfulness and verbal learning. Philadelphia: Lippincott, 1960.

\section{Notes}

1. A portion of the research and the preparation of this report was sponsored by Research Grant GB 213 from the National Science Foundation.

2. Present address: San Diego State College, San Diego, Calif. 